\title{
Human intestinal dendritic cells decrease cytokine release against Salmonella infection in the presence of Lactobacillus paracasei CNCM I-4034, a novel strain isolated from breast-fed newborns
}

\author{
M. Bermúdez-Brito ${ }^{1}$, S. Muñoz-Quezada ${ }^{1}$, C. Gómez-Llorente $^{1}$, E. Matencio ${ }^{2}$, M. J. Bernal ${ }^{2}$, \\ F. Romero ${ }^{2}$ and A. Gil ${ }^{1}$ \\ ${ }^{1}$ Department of Biochemistry and Molecular Biology II, Institute of Nutrition and Food Technology "José Mataix", \\ Biomedical Research Centre, University of Granada, Armilla, Granada, Spain and ${ }^{2}$ Hero Institute for Infant Nutrition, \\ Hero Spain, Alcantarilla, Murcia, Spain
}

\begin{abstract}
Dendritic cells (DCs) are antigen-presenting cells that are involved in immunity and tolerance. Innate pattern-recognition receptors, such as Toll-like receptors, play a crucial role in the host recognition of probiotics. Signalling via these receptors influences the chemokine and cytokine production, proteins that are essential in regulating immune response. Monocyte-derived dendritic cells (MoDCs) and murine DCs are different from human gut DCs; therefore, in this study, we used human DCs harvested from umbilical cord blood CD34+ progenitor cells, which are similar to the lamina propria DCs in the gut. In the present work, we investigated the immunomodulatory properties of a novel strain, Lactobacillus (L.) paracasei CNCM I-4034, isolated from exclusively breast-feeding infant feces, on human DCs against Salmonella (S.) typhimurium CECT 4594. Human intestinal DCs were directly challenged by addition of $L$. paracasei CNCM I-4034, S. typhimurium CECT 4594 or both. After 4 hours incubation, the medium was replaced with a new one, containing antibiotics and cytokines. After 20 hours, culture supernatants were collected for cytokine analysis. IFN- $\gamma$, IL-1 $\beta$, IL-6, IL-8, IL-10, IL-12p40, IL-12p70, TNF- $\alpha$, MCP-1(CCL2), MIP-1 $\alpha$ (CCL3), RANTES (CCL5), MDC (CCL22), IP-10 (CXCL10) and TGF- $\beta$ were measured by immunoassay, with a MILLIplex kit using the Luminex 200 system based in the xMap technology. Differences between treatments were assessed by the U Mann Whitney test. We found that DCs decreased the secretion of the pro-inflammatory cytokines, such as IL- 6 and IL-8, and chemokines, like MIP-1 $\alpha$ and RANTES, in response to stimulation with the probiotic and S. typhimurium. Interestingly, L. paracase $i$ was a potent inducer of TGF- $\beta 2$ secretion. In conclusion, $L$. paracasei CNCM I-4034 appears to be a promising modulator of immune system which is able to activate dendritic cells and reduce pathogen-induced inflammation.
\end{abstract}

